\title{
Regards sur l'expérience de la Commission de révision permanente des programmes au Québec
}

\author{
Marie-Pier Marchand \\ Université du Québec à Montréal \\ Astrid Brousselle \\ University of Victoria
}

\begin{abstract}
Résumé: Cet article s'intéresse à lexpérience de la Commission de révision permanente des programmes (CRPP) au Québec. À partir d'une revue documentaire, nous y proposons : 1) une description de la CRPP en présentant le contexte entourant sa création, sa démarche, le contenu de ses rapports ainsi que ses recommandations; et 2) un portrait de l'influence de la CRPP en explorant la prise en considération de ses recommandations au sein des décisions gouvernementales. Les constats qui se dégagent de notre démarche nous mènent à conclure que les recommandations de la CRPP nont pas été appliquées telles quelles, mais quelles ont probablement eu une influence ou un rôle symbolique au niveau des récentes coupes budgétaires entreprises par le gouvernement. Elles semblent également liées à l'institutionnalisation d'un nouveau mécanisme permanent de révision. En guise de discussion, nous soulevons différents questionnements, notamment la nature des travaux de la CRPP et larrimage entre les exercices de révision et la fonction évaluative au sein de lappareil gouvernemental.
\end{abstract}

Mots clés : examens des dépenses, dépenses publiques

\begin{abstract}
This paper focuses on the work done by the Commission de révision permanente des programmes (CRPP) in Quebec. Based on a literature review, we present: 1) a description of the CRPP including the context surrounding its creation, its approach, the content of its reports as well as its recommendations; and 2) a portrait of the influence of the CRPP by exploring whether or not government decisions have taken its recommendations into account. The findings that emerge from our analysis lead us to conclude that the recommendations of the CRPP have not fully been implemented, but that they had an influence or a symbolic role in relation to the recent budget cuts undertaken by the government. They also contributed to the institutionalization of a new permanent spending review mechanism. In our discussion, we raise a number of questions, including the nature of the work of the CRPP and the linkage between review exercises and the evaluative function within government.
\end{abstract}

Keywords: spending reviews, public expenditures

Corresponding author: Astrid Brousselle, University of Victoria, School of Public Administration, Human \& Social Development Building A302, 3800 Finnerty Road (Ring Road), Victoria, BC, V8P 5C2; courriel : astrid.brousselle@gmail.com 


\section{INTRODUCTION}

De nombreux pays ont fait récemment l'expérience de l'implantation de mécanismes de révision des dépenses publiques (Organisation for Economic Cooperation and Development, 2017). La finalité d'un exercice de révision des dépenses est essentiellement de fournir des propositions déconomies budgétaires (Arsenault, 2016) et sa popularité croît au sein des administrations étatiques déficitaires en quête d’espace budgétaire. Sur la scène québécoise, Jacob, Lamari, Poulin-Larivière et Beaucage (2016) ont d'ailleurs recensé différentes expériences de révision ayant été réalisées dans le passé, telles que les rapports Facal (Groupe de travail sur l'examen des organismes gouvernementaux, 1997), Boudreau (Secrétariat du Conseil du Trésor du Québec, 2005), Geoffrion (Secrétariat du Conseil du Trésor du Québec, 2006) et Rolland (Secrétariat du Conseil du Trésor du Québec, 2007). En outre, plus récemment s'achevaient les travaux de la Commission de révision permanente des programmes (CRPP) mise en place par le gouvernement de Philippe Couillard afin de dégager un surplus budgétaire.

La CRPP est une entité dont le mandat s’inscrit dans un exercice évaluatif sommatif, s'appuyant sur les données existantes, afin de guider le gouvernement pour qu'il dégage une marge de manœuvre budgétaire, laquelle ne peut être obtenue qu'en recourant à des coupures importantes (Arsenault, 2016). Bien que la CRPP mette l'accent principalement sur le mécanisme de révision, l'activité propre à l'évaluation de programme, qui comprend quant à elle une démarche de collecte et d'analyse de données qui découle ultimement sur la formulation d'un jugement sur la valeur d'un programme (Secrétariat du Conseil du Trésor du Québec, 2013), s’y trouve subordonnée (Jacob et coll., 2016). Du coup, l'influence de la CRPP et son rôle au sein de l'appareil gouvernemental demeurent une question d'intérêt et interpellent spécifiquement la communauté des évaluateurs.

Lobjectif de cet article est de poser un regard sur l'expérience de la CRPP au Québec, tant en matière de réalisations que de ses impacts. Pour ce faire, nous avons adopté une approche exploratoire et descriptive dans le but de présenter la CRPP et son contexte de réalisation ainsi que de dresser un portrait de son influence sur les mesures gouvernementales entreprises. En plus de préciser les objectifs et la nature de notre démarche, les sections qui suivent fournissent une description de la CRPP et de son influence érigée à partir d’une revue documentaire et proposent une discussion faisant état des constats et questionnements résultant de nos travaux.

\section{OBJECTIFS ET DÉMARCHE}

La démarche que nous avons entamée poursuit deux objectifs. Le premier objectif consiste à proposer une description sommaire de la CRPP, du contexte de sa création jusquà la fin de son mandat. Le second objectif est quant à lui en lien avec l'exploration de l'influence de la CRPP, aspirant à recenser les différentes décisions et coupes budgétaires qui en ont résulté.

Différentes sources de données ont été identifiées et étudiées afin d'atteindre ces deux objectifs : 1) les différents rapports de la CRPP; 2) la littérature grise, 
incluant des présentations en format PowerPoint en lien avec la CRPP, les comptes publics diffusés par le gouvernement du Québec et les décisions budgétaires prises entre 2014 et 2016; 3) la documentation officielle du gouvernement en lien avec le processus de révision; et 4) les articles de presse publiés dans deux journaux majeurs francophones entre 2014 et 2016 (La Presse et Le Devoir), traitant de la CRPP (une liste exhaustive des articles consultés est disponible auprès des auteurs, sur demande). Plus précisément, à partir de la base de données Eureka, nous avons effectué une recherche avancée par mots clés ("rapport Robillard", " commission révision programme ", "Claude Montmarquette »), en couvrant les publications parues entre 2014 et 2016 et en sélectionnant La Presse, La Presse+, Le Devoir ainsi que le Devoir (site Web) à titre de sources de provenance. Les résumés des articles issus de cette recherche ont été lus afin de ne retenir que les articles pertinents traitant de la CRPP. Un répertoire totalisant 112 articles de presse a été créé, lequel a été analysé dans son entièreté. Mentionnons que l’analyse sétend aux publications de 2016 afin de couvrir l’année qui suit la fin des travaux de la CRPP. N’aspirant pas à contraster des idées et mettre en parallèle des perspectives divergentes, mais plutôt à recenser les réactions et les mesures qui ont fait suite aux recommandations de la CRPP, nous n’avons pas jugé essentiel détendre la recherche aux journaux anglophones québécois. L'adéquation de ce choix a d'ailleurs été validée, car nous avons observé une grande redondance d'informations dans les deux journaux consultés, confirmant le fait qu'il est peu probable que nous ayons manqué des informations importantes en excluant les journaux anglophones. En somme, l'analyse croisée des décisions budgétaires prises entre 2014 et 2016, des rapports consultés et des articles de journaux permet d'avoir un portrait relativement complet des mesures gouvernementales ayant découlé de la CRPP.

Nous voulions également documenter l'expérience de la CRPP à partir de témoignages de divers acteurs et experts œuvrant au sein de la fonction publique et ayant été concernés par ses travaux. En raison d’enjeux politiques importants au moment de notre collecte de données, il a été impossible de recruter des participants désireux d'aborder le sujet, tant auprès des membres de la CRPP qu'auprès d'acteurs en poste dans l'administration gouvernementale. Nous avons entamé une phase de recrutement au préalable afin de vérifier la possibilité de réaliser des entretiens. Au total, six tentatives formelles ont été entreprises (prises de contact directes et prises de contact indirectes par l'entremise d'informateurs clés), lesquelles ont toutes résulté en des refus, des abstentions ou nont pas obtenu de réponse de la part des personnes sollicitées. Devant cette situation et ces difficultés de recrutement, nous avons décidé d'écarter cet objectif de décrire l'expérience de la CRPP à partir de témoignages. Soulignons toutefois que nous avons tenu certaines discussions informelles avec des acteurs ayant été impliqués dans l'exercice de la CRPP, ce qui nous a permis de valider plusieurs observations tirées des écrits consultés.

La section subséquente présente le fruit de nos analyses. Dans un premier temps, une description sommaire de la CRPP est proposée, traitant du contexte entourant sa création, la démarche qu'elle a empruntée, le contenu des rapports qu'elle a publiés ainsi que les principales recommandations quelle a formulées. 
En second lieu, nous explorons l'influence de la CRPP, en exposant les différentes décisions et coupes budgétaires subséquentes et relatives à la CRPP que nous avons recensées.

\section{PORTRAIT DE LA CRPP}

\section{Le contexte d'implantation}

En 2014, le gouvernement du Québec, alors nouvellement élu, mettait sur pied deux commissions indépendantes de l'appareil gouvernemental, la Commission de révision permanente des programmes (CRPP), dirigée par Lucienne Robillard, et la Commission d'examen sur la fiscalité québécoise, sous la direction de Luc Godbout. Les deux commissions devaient travailler en complémentarité afin que le gouvernement atteigne ses cibles budgétaires (Gouvernement du Québec, 2014c).

Plus spécifiquement, la création de la CRPP s'inscrivait dans le respect des engagements préélectoraux annoncés par le Parti Libéral du Québec qui prévoyait mettre en place une structure rigoureuse de gestion des dépenses afin « d'offrir un répit aux familles de la classe moyenne » (Parti Libéral du Québec, 2014, p. 44), aspirant ainsi à dégager un surplus budgétaire permettant de réduire le fardeau de la dette provinciale et d'annoncer des diminutions d'impôts, notamment par l'abolition de la taxe santé et l'indexation de la tarification des services de garde (Parti Libéral du Québec, 2014).

Le mandat de la CRPP était de " proposer la mise en place d'un mécanisme permanent de révision des programmes » ainsi que de «formuler des recommandations concernant les programmes à réviser» (Gouvernement du Québec, 2014c, p. 5). Lobjectif ultime de sa création visait à orienter l'action gouvernementale de façon à atteindre certaines " cibles budgétaires » et à retrouver une " marge de manœuvre » (Gouvernement du Québec, 2014c, p. 5). Les journaux indiquent que les Commissions Robillard et Godbout « devront identifier des moyens de dégager un espace financier de 3,2 milliards \$ à temps pour le prochain budget du Québec ", un an plus tard (Teisceira-Lessard, 2014); la visée finale étant d'atteindre, pour l'année financière 2015-2016, l'équilibre budgétaire (Arsenault, 2014; Lessard et Teisceira-Lessard, 2014).

Les travaux de la CRPP ont été entamés le 11 juin 2014 et se sont achevé le 31 août 2015, date à laquelle se terminait le mandat confié par le gouvernement. Nommés par le Conseil du Trésor, les membres de la CRPP présentaient des expertises et des parcours variés (sphère politique, domaine universitaire, appareil gouvernemental, sciences économiques, analyse et évaluation de programmes, administration, etc.) (Gouvernement du Québec, 2014c). Plus spécifiquement, sous la présidence de Lucienne Robillard, ancienne ministre fédérale sous le gouvernement libéral, les travaux de la CRPP étaient appuyés par Claude Montmarquette et Robert Gagné, économistes et professeurs-chercheurs notamment affiliés au Centre interuniversitaire de recherche en analyse des organisations (CIRANO), ainsi que Michèle Bourget et Mireille Fillion, ex-administratrices 
détat (Jacob et coll., 2016). Mentionnons également que les membres de la CRPP ont été soutenus par différentes personnes-ressources lors de leurs travaux (Gouvernement du Québec, 2014c).

Il importe de préciser quau moment de la création de la CRPP, un mécanisme dévaluation des programmes est déjà en place au sein de l'appareil gouvernemental. En effet, en date du 4 juin 2013, le gouvernement du Québec adoptait sa Directive concernant lévaluation de programme dans les ministères et organismes, laquelle avait pour visée principale de «favoriser lévaluation de programme au sein de l'Administration gouvernementale et de renforcer les saines pratiques de gestion et la gestion axée sur les résultats » (Gouvernement du Québec, 2014b). Cette directive amenait donc les ministères et les organismes à rendre compte au Secrétariat du Conseil du Trésor du Québec des exercices d’évaluation de leurs programmes.

Plus spécifiquement, cette orientation stipule qu'un responsable de lévaluation des programmes doit être nommé au sein de chaque ministère et organisme assujetti à la Loi sur l'administration publique (Gouvernement du Québec, 2014b). Ce responsable doit élaborer un plan pluriannuel d'évaluation, dans lequel sont précisés les programmes à être évalués, certains étant identifiés sur la base de besoins exprimés par l'organisation et d'autres étant ciblés par un décret ou une demande gouvernementale (Gouvernement du Québec, 2014b). Pour chaque évaluation ensuite réalisée, un cadre dévaluation doit être fourni au Secrétariat du Conseil du Trésor du Québec, en plus des résultats et recommandations émanant de la démarche évaluative (Gouvernement du Québec, 2014b). Certaines des recommandations formulées par la CRPP reposent sur les données issues de ce mécanisme d'évaluation en fonction dans les ministères.

\section{La démarche}

La démarche de la CRPP supportait une approche à la fois ministérielle et interministérielle, c'est-à-dire que les différents ministères ont été interpellés afin de participer à l'exercice de révision des programmes à l'interne, en plus dêtre invités à prendre part aux tables horizontales interministérielles créées dans le but d'examiner la cohérence et le chevauchement des interventions entre ministères (Gouvernement du Québec, 2014c). Dans le cadre de ses travaux, la CRPP a également promu une culture de dialogue social en instaurant une tribune Web accessible par les citoyens et regroupements et en invitant certaines associations patronales et syndicales d'importance ainsi que les gestionnaires et employés de la fonction publique à exprimer leurs positions et propositions quant aux programmes gouvernementaux (Gouvernement du Québec, 2014c).

La CRPP mentionne avoir retenu trois critères pour l'analyse des programmes à réviser : 1) la pertinence; 2) l'efficacité; et 3) l'efficience (Gouvernement du Québec, 2014c). Le Tableau 1 présente la définition de ces critères, tels que le propose la CRPP. Elle affirme par ailleurs accorder une importance particulière aux personnes vulnérables, notamment sur le plan des impacts de ses recommandations sur ces dernières. 
Tableau 1. Les critères de la CRPP pour l'analyse des programmes (Gouvernement du Québec, 2014c, p. 29)

\begin{tabular}{|c|c|}
\hline Critères & Définitions \\
\hline Pertinence & $\begin{array}{l}\text { Un programme pertinent est: } \\
\text { - Un programme dont les objectifs correspondent aux } \\
\text { besoins des clientèles ainsi qu'aux priorités de l'État; } \\
\text { - Un programme qui aurait pu être créé dans le contexte actuel; } \\
\text { - Un programme dont l'abolition partielle ou totale aurait } \\
\text { des conséquences négatives importantes sur les plans social, } \\
\text { économique et environnemental. }\end{array}$ \\
\hline Efficacité & $\begin{array}{l}\text { Un programme efficace est: } \\
\text { - Un programme s'adressant à des clientèles bien définies et } \\
\text { répondant à des objectifs clairs et mesurables; } \\
\text { - Un programme atteignant les objectifs pour lesquels il a } \\
\text { été créé; } \\
\text { - Un programme cohérent avec les autres programmes existant } \\
\text { dans le même domaine d'intervention. }\end{array}$ \\
\hline Efficience & $\begin{array}{l}\text { Un programme efficient est: } \\
\text { - Un programme qui ne se dédouble pas avec d'autres } \\
\text { programmes; } \\
\text { - Un programme dont il n'est pas possible d'atteindre les résultats } \\
\text { par des solutions moins coûteuses; } \\
\text { - Un programme dont la gouvernance, les processus administratifs } \\
\text { et les modes de financement et de livraison peuvent être } \\
\text { considérés comme optimaux. }\end{array}$ \\
\hline
\end{tabular}

Les travaux de la CRPP s'appuient sur l'analyse de divers documents :

- des évaluations de programmes effectuées par les ministères;

- des études produites à lexterne, entre autres par des comités mandatés par le gouvernement;

- des analyses des organismes de recherche en politiques publiques;

- des analyses comparatives avec les programmes mis en place dans d’autres pays (Gouvernement du Québec, 2014c, p. 27).

Les travaux de la CRPP constituent ainsi une analyse secondaire de rapports existants. À cet égard, la CRPP souligne qu'elle trouve que « l'information concernant plusieurs programmes est déficiente et peu de programmes sont systématiquement évalués » (Gouvernement du Québec, 2014c, p. 31).

\section{Le contenu des rapports publiés}

Le premier rapport de la CRPP, déposé en novembre 2014, présente la nature de son mandat, aborde les principes et critères à la base de sa démarche et présente 
ses constats initiaux (Gouvernement du Québec, 2014c). La CRPP y fait état de ses premières recommandations relativement à divers sujets et programmes : l'aide financière aux municipalités, la Financière agricole du Québec et le Programme d'assurance stabilisation des revenus agricoles, le Programme de places à contribution réduite dans les services de garde éducatifs, le financement des étudiants universitaires internationaux, les services ambulanciers, le budget discrétionnaire des ministres, le Programme de soutien à l'action bénévole et le financement public des écoles privées (Gouvernement du Québec, 2014c).

Un second rapport est remis en juin 2015, lequel se décline en cinq sousrapports, incluant un rapport sommaire (Gouvernement du Québec, 2015a) et quatre volumes. Le premier de ces volumes s'intéresse aux fondements à la base d'un État performant et discute plus spécifiquement du mécanisme de révision permanente des programmes (Gouvernement du Québec, 2015b). Le second volume expose quant à lui quelques pistes de révision relativement à certains programmes gouvernementaux (Gouvernement du Québec, 2015c). Le troisième volume présente les travaux de la CRPP en ce qui a trait à l'examen du modèle de révision des programmes effectif au sein du Gouvernement du Canada ainsi qu'une étude comparative réalisée par l'Observatoire de l'administration publique de l'École nationale d'administration publique (ENAP) s'intéressant aux pratiques évaluatives de différents pays de l'Organisation de coopération et de développement économiques (OCDE) (Gouvernement du Québec, 2015d). Finalement, le quatrième volume détaille les résultats d'une étude réalisée par la firme Deloitte en ce qui a trait aux efforts de la Table sur l'aide financière au développement économique visant à brosser un portrait de la situation du Québec relativement au développement économique, à comparer cette situation à différentes juridictions similaires et à identifier des pistes d'amélioration (Gouvernement du Québec, 2015e).

\section{Les recommandations}

Les premières recommandations formulées par la CRPP visent à guider le gouvernement à faire des choix de coupes budgétaires et à poser les bases pour l'instauration d'un système de révision permanente des programmes. Les recommandations émises à cet effet concernent, entre autres, la mise en place d'une culture évaluative continue et systématique au service du bien commun et de la population, au sein d'un état qui se doit d'être transparent, stratège, innovant et en harmonie avec ses différents tentacules ministériels (Gouvernement du Québec, 2015c). La CRPP suggère par ailleurs d'intégrer un système de révision permanente des programmes à la structure administrative; un système transparent, indépendant et opéré par des acteurs compétents (Gouvernement du Québec, 2015c).

La CRPP a également étudié l'approche gouvernementale interministérielle en place. Elle juge de l'importance de conserver l'approche des tables horizontales en la pérennisant et en facilitant la diffusion des travaux effectués par les différentes tables, c'est-à-dire la Table sur l'aide financière au développement 
économique, la Table sur la présence internationale du Québec, la Table sur la culture, la Table sur l'aide aux municipalités et la Table sur l'emploi et la formation. La CRPP recommande entre autres l'intensification ou l'élargissement des travaux de certaines de ces tables et propose à cet égard quelques pistes d'améliorations et de réflexion (Gouvernement du Québec, 2015c).

Les recommandations suivantes de la CRPP portent sur différents secteurs de dépenses, les principales étant décrites au sein du Tableau 2.

Après l'identification de ces pistes de coupes budgétaires ciblées par la CRPP, nous avons tenté d'explorer l'influence de la CRPP en analysant la prise en considération de ses recommandations au sein des mesures gouvernementales. À la suite de notre analyse, il apparaît que cette influence s'observe au niveau de la mise en œuvre d'un mécanisme permanent de révision et au niveau des coupes budgétaires réalisées par le gouvernement; deux sphères directement reliées au mandat en deux volets confiés à la CRPP.

\section{INFLUENCE DE LA CRPP}

\section{Instauration d'un mécanisme permanent de révision}

Le premier volet du mandat de la CRPP visait à « proposer la mise en place d'un mécanisme permanent de révision des programmes " (Gouvernement du Québec, 2014c, p. 5). Les travaux de la CRPP ont spécifiquement mis en évidence

Tableau 2. Principales recommandations de la CRPP en fonction des différents secteurs de dépenses

- Recommandation de la CRPP : réduire les transferts financiers versés par le gouvernement et encourager les municipalités à exercer un meilleur contrôle de leurs dépenses, notamment en modulant les dispositions ré-

Aide financière aux municipalités glementaires actuelles de façon à ce qu'elles contrôlent mieux la rémunération de leurs employés (Gouvernement du Québec, 2014c, p. 142-142).

- Les municipalités devraient absorber plus de la moitié des coupes (Dutrisac, 2014; Lessard, 2014).

- La CRPP suggère de transférer la responsabilité de la prime de risque aux membres assurés, suggérant de cesser de financer le programme sous sa forme actuelle

Programme d'aide pour les agriculteurs (Programme d'assurance stabilisation des revenus agricoles) et de diminuer l'aide financière versée à la Financière agricole du Québec (Gouvernement du Québec, 2014c, p. 143).

- La CRPP encourage le gouvernement à revoir son Programme de crédit de taxes foncières agricoles, notamment en le structurant davantage et en révisant ses critères d'admissibilité (Gouvernement du Québec, 2015c). 
Tableau 2. Continué

\begin{tabular}{|c|c|}
\hline $\begin{array}{l}\text { Programme de places } \\
\text { à contribution réduites } \\
\text { dans les services de } \\
\text { garde éducatifs }\end{array}$ & $\begin{array}{l}\text { - La CRPP recommande d'améliorer la gestion des services } \\
\text { de garde, en proposant le financement selon la présence } \\
\text { réelle, en instaurant le ratio d'une éducatrice pour dix } \\
\text { enfants et en privilégiant le coût de revient réel afin de } \\
\text { déterminer les subventions versées (Gouvernement du } \\
\text { Québec, 2014c, p. } 144 \text { à 147). } \\
\text { - La CRPP propose de réviser le système de financement } \\
\text { des garderies subventionnées, soutenant une augmen- } \\
\text { tation de la tarification et une bonification du crédit } \\
\text { d'impôt pour frais de garde, et suggère au gouvernement } \\
\text { de suspendre la création de nouvelles places (Gouverne- } \\
\text { ment du Québec, 2014c, p. } 144 \text { à 147). }\end{array}$ \\
\hline Services ambulanciers & $\begin{array}{l}\text { - La CRPP recommande la révision de l'ensemble des } \\
\text { contrats avec les entreprises privées afin d'en améliorer } \\
\text { la gouvernance et la gestion et de changer la tarifica- } \\
\text { tion en vigueur, notamment pour les personnes âgées } \\
\text { (Gouvernement du Québec, 2014c, p. 149). }\end{array}$ \\
\hline \multirow[t]{3}{*}{ Secteur de l'éducation } & $\begin{array}{l}\text { - La CRPP suggère de moduler et de dérèglementer le } \\
\text { mode de financement pour les étudiants universitaires } \\
\text { internationaux en instaurant de nouveaux quotas pour } \\
\text { les étudiants bénéficiant d'exemptions (Gouvernement } \\
\text { du Québec, 2014c, p. 148). }\end{array}$ \\
\hline & $\begin{array}{l}\text { - La CRPP invite le gouvernement à étudier de façon plus } \\
\text { approfondie la question du transfert des clientèles avant } \\
\text { d'entamer une diminution des subventions au secteur } \\
\text { privé (Gouvernement du Québec, 2014c). Elle conclut } \\
\text { plus tard en affirmant qu'aucune économie ne résulterait } \\
\text { d'une telle diminution, soutenant du coup le maintien } \\
\text { des deux secteurs (Gouvernement du Québec, 2015c). }\end{array}$ \\
\hline & $\begin{array}{l}\text { - Les recommandations de la CRPP, si elles étaient appli- } \\
\text { quées, se traduiraient par une réduction du budget d'un } \\
\text { milliard de dollars, soit environ } 9 \% \text { du budget global } \\
\text { (Chouinard, 2014; Lessard, 2014). }\end{array}$ \\
\hline $\begin{array}{l}\text { Budgets discrétionnaires } \\
\text { des ministres }\end{array}$ & $\begin{array}{l}\text { - La CRPP recommande de les abolir (Gouvernement du } \\
\text { Québec, 2014c). }\end{array}$ \\
\hline $\begin{array}{l}\text { Programme de soutien à } \\
\text { l'action bénévole }\end{array}$ & $\begin{array}{l}\text { - La CRPP recommande d'y mettre fin (Gouvernement du } \\
\text { Québec, 2014c). }\end{array}$ \\
\hline $\begin{array}{l}\text { Société des alcools du } \\
\text { Québec }\end{array}$ & $\begin{array}{l}\text { - La CRPP critique son monopole et suggère une li- } \\
\text { béralisation du commerce des vins et spiritueux } \\
\text { (Gouvernement du Québec, 2015c). }\end{array}$ \\
\hline Administration fiscale & $\begin{array}{l}\text { - La CRPP conclut qu'il importe au gouvernement de } \\
\text { veiller à la performance de Revenu Québec et encour- } \\
\text { age ce dernier à réfléchir sur l'éventualité d'un transfert } \\
\text { des activités fiscales au palier fédéral (Gouvernement du } \\
\text { Québec, 2015c). }\end{array}$ \\
\hline
\end{tabular}


Tableau 2. Continué

\begin{tabular}{ll}
\hline & - La CRPP est d'avis que le gouvernement devrait confier \\
& la gestion du programme à un ministère, lequel devrait \\
& s'assurer de dresser un portrait de la clientèle que le \\
& programme dessert (Gouvernement du Québec, 2015c). \\
Programme de & La CRPP suggère de rendre moins complexe la démarche \\
perception des & d'exemption au programme et d'imposer une tarifica- \\
pensions alimentaires & tion pour la perception des pensions dans le cas où le \\
& programme comprendrait une adhésion automatique \\
& (Gouvernement du Québec, 2015c). \\
\hline - La CRPP a intenté l'examen de la question de l'accessibilité \\
aux services et constate à cet égard la nécessité pour le \\
gouvernement de considérer la pertinence des soins \\
en fonction d'un plan d'action établi, d'appliquer le \\
mode de financement axé sur le patient et de favoriser \\
l'interdisciplinarité dans la prestation des soins de première \\
ligne (Gouvernement du Québec, 2015c).
\end{tabular}

l'importance de la présence d'un tel processus afin d'assurer la performance des programmes (Gouvernement du Québec, 2015c. Suivant cette recommandation, le gouvernement du Québec a instauré en septembre 2015 le Bureau de la révision permanente des programmes, exigeant du coup une démarche rigoureuse dévaluation et de révision pour le renouvellement des programmes (Portail Québec, 2015. Le gouvernement a ensuite procédé à une restructuration en abolissant cette entité et en publiant en mars 2017 sa Stratégie de gestion des dépenses. Budget de dépenses 2017-2018, dans laquelle est décrite la culture de révision permanente souhaitée par ce dernier (Gouvernement du Québec, 2017). Dans ce document, la révision des programmes est associée au renforcement de " la viabilité et la qualité des interventions gouvernementales en fonction des besoins prioritaires et des ressources disponibles ", permettant ainsi " une allocation optimale des ressources de l'État » (Gouvernement du Québec, 2017, p. 13). Cette culture de révision est instaurée par le gouvernement afin de mieux encadrer la prise de décisions et de veiller à la performance des programmes (Gouvernement du Québec, 2017).

Plus spécifiquement, il apparaît que le mécanisme prôné se positionne en complémentarité avec la planification annuelle des dépenses et la Directive concernant lévaluation de programme dans les ministères et organismes adoptée en 2013 (Gouvernement du Québec, 2017). Ce mécanisme de révision permanente se concrétise par un examen stratégique des programmes et a pour objectifs :

- détablir un diagnostic à propos du rendement d'un organisme ou d'un programme, laissant ainsi une grande place à lévaluation de programme;

- de déterminer les actions requises en vue d’optimiser ou d’abolir un programme; 
- de planifier la mise en œuvre, par l’organisation concernée, des solutions retenues et le suivi systématique des résultats attendus (Secrétariat du Conseil du Trésor du Québec, 2017).

L’examen stratégique vise les programmes moins performants identifiés par les ministères et organismes, en partenariat avec le Secrétariat du Conseil du Trésor du Québec, et peut s'intéresser à différentes dimensions de l'intervention gouvernementale : 1) un modèle d'affaires ou une fonction administrée par plusieurs ministères ou organismes; 2) un système de production d'un bien ou d'un service public; ou 3) une dépense (Gouvernement du Québec, 2017, p. 14-15). Pour cet examen, les mêmes critères que ceux de la CRPP, c'est-à-dire la pertinence, l'efficacité et l'efficience, sont mis de l'avant (Gouvernement du Québec, 2017).

\section{Coupes budgétaires}

Le second volet du mandat de la CRPP aspirait à " formuler des recommandations concernant les programmes à réviser » dans le but de créer un espace budgétaire (Gouvernement du Québec, 2014c, p. 5). Les résultats de notre démarche indiquent que le gouvernement a procédé à des coupes importantes dans les programmes publics, ce qui a permis d’obtenir un surplus budgétaire. En effet, les états financiers du Québec indiquent des surplus pour les années financières 20142015 et 2015-2016 (Gouvernement du Québec, 2016), alors que la province était avec des déficits annuels en 2012-2013 et 2013-2014 (Gouvernement du Québec, 2014a). Toutefois, les mesures adoptées en ce sens n'apparaissent pas directement rattachées aux recommandations de la CRPP. Jacob et coll. (2016) soulignent que les recommandations de la CRPP ne sont pas appliquées telles quelles ou ne sont appliquées que partiellement, mais que de nombreux secteurs d'activités voient leur financement considérablement réduit.

Un chercheur de l'Institut de recherche et d'informations socio-économiques (IRIS) a recensé les coupes annoncées dans les quotidiens et les hebdomadaires au sein d'un document de réflexion (Nguyen, 2016). Il identifie, entre 2014 et 2016, 342 compressions qui équivalent à 4 milliards de dollars. Il s'agit là d'une mesure imparfaite, puisque certaines mesures ont pu être annoncées puis modifiées, et que les mesures ne concernent pas seulement celles imposées par le gouvernement du Québec, mais l'ensemble des restrictions budgétaires des organismes provinciaux et fédéral (Lévesque, 2015). Les données de ce rapport demeurent intéressantes pour indiquer les secteurs touchés par les coupes du gouvernement (voir Tableau 3).

À titre d'exemple, le secteur de l'éducation se voit amputé d'une aide totalisant 372,8 millions de dollars alors que le budget pour la santé et les services sociaux est réduit de 963,4 millions de dollars (Nguyen, 2016). L'aide sociale, l'accès au logement ainsi que différentes organisations et campagnes de sensibilisation pour contrer le sexisme, l'homophobie et les inégalités homme/femme sont également sujets à des compressions, respectivement de lordre de 65 millions de dollars, 53 millions et 2,718 millions de dollars (Nguyen, 2016). 
Tableau 3. Recensement des différentes coupes effectuées par le gouvernement entre 2014 et 2016 par secteurs d'activités (Nguyen, 2016, p. 5)

\begin{tabular}{lc}
\hline Secteurs d'activités & Nombres decoupes \\
\hline Éducation primaire et secondaire & 77 \\
Éducation postsecondaire & 65 \\
Santé et services sociaux & 78 \\
Emploi et solidarité sociale & 6 \\
Fonction publique & 17 \\
Famille & 15 \\
Développement local et régional & 17 \\
Logement et habitation & 9 \\
Environnement & 4 \\
Égalité homme/femme, sensibilisation & 8 \\
contre le sexisme et l'homophobie & 4 \\
Justice & 18 \\
Culture & 3 \\
Transport & 12 \\
Hausse de tarif & 342 \\
Total & \\
\hline
\end{tabular}

La revue de presse réalisée indique que les recommandations de la CRPP ont souvent été perçues comme politiquement inapplicables, y compris par plusieurs instances et membres du gouvernement, ce qui expliquerait quelle ne semble pas avoir eu une influence directe sur le choix des programmes coupés. En effet, une fois les rapports de la CRPP publiés, les recommandations ont fait lobjet de nombreuses critiques non seulement en provenance des différents partis politiques, des centrales syndicales, des parties concernées par les coupes, mais également de la part de différents ministres. Ainsi, les recommandations de la CRPP ne sont pas directement liées aux coupes observées, mais elles ont toutefois certainement alimenté le débat public et politique.

\section{DISCUSSION}

La mission de la CRPP était notamment d'identifier des sources d'économies gouvernementales dans un agenda de réduction des dépenses publiques. Entre 2014 et 2016, le gouvernement a coupé de façon transversale dans différents secteurs, particulièrement dans léducation et la santé et les services sociaux, sans toutefois que le choix des mesures puisse être directement lié aux recommandations de la CRPP. Néanmoins, le gouvernement sest appuyé sur ses rapports pour justifier la nécessité de procéder à des coupes dans de nombreuses sphères d'activité. De façon générale, notre démarche nous porte à conclure que la CRPP a contribué au débat sur la nature des coupes budgétaires et qu'elle a joué un rôle symbolique 
dans un exercice prévu de rationalisation des dépenses publiques. Somme toute, l'exercice semble avoir atteint ses objectifs relatifs à l'espace budgétaire à créer, les états financiers subséquents à la CRPP en faisant foi.

Notre analyse soulève de nombreux questionnements concernant la nature du processus de révision entamé par la CRPP. D’abord, l'agenda était très ambitieux. En deux ans, huit programmes majeurs devaient être examinés en regard des critères de pertinence, d’efficacité et d'efficience (Gouvernement du Québec, 2014c), ce qui demeure une contrainte de temps importante étant donnél'ampleur de la tâche.

Ensuite, mentionnons que la CRPP a dû faire face, comme elle le souligne elle-même (Gouvernement du Québec, 2015c, p. 7, 19, 72), à un manque de données fiables ou actuelles. Cette situation nous semble préoccupante compte tenu du fait que la présence d'informations de qualité constitue une condition de réussite à tout exercice de révision des programmes (Arsenault, 2016). En particulier, lexercice de la CRPP soulève la question de l'influence des évaluations gouvernementales dans le processus de révision : de quelles façons ces rapports ont-ils été utilisés et utiles à l'exercice de révision? Ce questionnement met également en exergue la situation liée à l'arrimage entre la fonction évaluative au sein du gouvernement, le rôle temporaire attribué à la CRPP ainsi que celui d'un mécanisme de révision permanente.

Par ailleurs, alors que la CRPP indiquait vouloir effectuer une révision systématique selon les critères de pertinence, d'efficacité et d'efficience, les rapports ne présentent aucune discussion selon les critères retenus. La transparence et la systématicité de l'exercice se révèlent ainsi questionnables. Par ailleurs, le critère d'efficacité retenu est très spécifique à l'exercice. La CRPP définit un programme efficace comme étant « un programme s’adressant à des clientèles bien définies et répondant à des objectifs clairs et mesurables; un programme atteignant les objectifs pour lesquels il a été créé; un programme cohérent avec les autres programmes existants dans le même domaine d'intervention » (Gouvernement du Québec, 2014c, p. 29). L’utilisation d'une telle définition soulève quelques interrogations. Que faire si le programme a une couverture plus grande que celle qui était prévue à l'origine? Comment intégrer les effets inattendus? Dans une optique plus large, comment mesurer l'efficacité des programmes en éducation et en santé, par exemple, quand ceux-ci ont des effets diffus, multiples et à long terme? Ces programmes pourraient être, dès le départ, désavantagés par l'application d'une telle logique évaluative dans un exercice de révision budgétaire.

En outre, la question de l'impact sur les personnes les plus vulnérables est souvent éludée. Alors que la CRPP disait vouloir minimiser l'impact sur les populations vulnérables, elle demeure très timide concernant la formulation de propositions concrètes. Certaines recommandations sont accompagnées de la suggestion de diriger les bénéficiaires vers d'autres programmes normés, mais sans décrire comment pourrait s'opérationnaliser cette démarche. Au-delà de cette recommandation, aucune analyse d'impact des coupes suggérées sur les plus 
vulnérables n'est effectuée et aucune mesure d'atténuation n'est proposée. Nous en venons également à un constat similaire quant au dialogue social prôné en amont par la CRPP. À nouveau, bien que cette intention de donner une voix aux différentes parties prenantes soit louable, il est difficile de percevoir de quelle façon ce dialogue a été pris en considération, les rapports n'y faisant que très peu référence.

Nous désirons également soulever différentes interrogations en lien avec l'intégration de la CRPP à l'appareil gouvernemental, alors qu’il existe déjà un mécanisme institutionnalisé pour l'évaluation des programmes publics. Le mandat de la CRPP s'inscrivait au départ comme un exercice ponctuel avec un objectif budgétaire spécifique. Il existe des différences non négligeables entre les fonctions de révision budgétaire, aspirant davantage à des finalités sommatives et les fonctions d'évaluation dans l'appareil gouvernemental, entretenant davantage des visées formatives. En effet, alors que la révision budgétaire vise essentiellement à « produire des propositions d'économies ", lévaluation, dans son institutionnalisation, a pour rôle de « fournir aux dirigeants des analyses pour améliorer l'efficience et l'efficacité de leurs interventions » (Arsenault, 2016, p. 92). Étant donné la complémentarité de ces deux fonctions et leur implantation dans l'appareil gouvernemental, n'y a-t-il pas lieu de se questionner sur les possibles chevauchements et duplications des rôles et mandats de la CRPP et des activités évaluatives déjà en place au sein des ministères? La venue des travaux de la CRPP a-t-elle été accompagnée d'un partage clair des responsabilités et tâches à effectuer? Le mandat de la CRPP a-t-il permis une collaboration entre les acteurs concernés ou, au contraire, a-t-il été perçu par certains comme une menace ou une entité dont les fonctions nont pas été bien précisées et définies? Comment les principaux acteurs touchés par cette initiative ont-ils accueilli et vécu l'exercice? Étant donné l'impossibilité de discuter avec des personnes ayant participé d'une manière ou d'une autre à l'exercice de révision, nous n'avons pu trouver réponse à ces questions pourtant centrales.

En continuité, il appert qu'il serait légitime de mettre en doute le système récemment mis en place par le gouvernement relativement à la révision permanente des programmes. Ce nouveau mécanisme ne se veut plus un exercice temporaire comme l'expérience de la CRPP, mais bien une pratique institutionnalisée à l'appareil gouvernemental et entrant en complémentarité avec la fonction évaluative déjà en vigueur au sein des ministères et organismes. Tel que suggéré par la CRPP, le mécanisme devra être transparent, indépendant et piloté par des acteurs compétents (Gouvernement du Québec, 2015c). L'implantation d'une nouvelle procédure exige bien souvent un certain temps avant que ses rouages parviennent à fonctionner adéquatement et efficacement, nous en avons bien conscience. Néanmoins, il sera d'un grand intérêt pour la communauté de suivre de près les travaux réalisés en ce sens. Cela ne va pas sans rappeler les questionnements que nous avons soulevés plus tôt quant à la qualité et à la pertinence des données disponibles provenant des exercices évaluatifs conduits au sein des ministères et organismes. De récents travaux de Smits et Jacob (2014) quant à la fonction évaluative dans l'administration publique québécoise soulignent d'ailleurs la faible présence de mesures de soutien de la pratique de la part des ministères centraux 
ainsi que des lacunes au niveau de la transparence dans le processus auprès des citoyens.

\section{CONCLUSION}

Une bonne gestion publique implique de trouver un moyen de sassurer que les programmes publics sont ceux qui servent le mieux les intérêts des citoyens. L'exercice de la CRPP au Québec soulève beaucoup de questions, sur la nature de la démarche, la recevabilité de ses recommandations et l'institutionnalisation d'un mécanisme de révision permanente au sein de l'appareil gouvernemental qui dispose déjà d’une capacité évaluative de ses programmes. Les objectifs de notre démarche étant de documenter l'expérience de la CRPP ainsi que son influence, les constats que nous avons réalisés nous mènent à affirmer qu'il sera impératif de veiller à la qualité du mécanisme permanent de révision nouvellement mis en place par le gouvernement, car des décisions budgétaires entrainant des coupes importantes dans différents programmes ou secteurs d’activités en résultent inévitablement.

Finalement, nous partageons certaines craintes relativement à l'opacité qui semble avoir entouré l'expérience de la CRPP, situation sans aucun doute reliée aux refus et abstentions auxquels nous avons fait face lors de notre phase de recrutement de participants à interroger. Nous pensons que la capacité de discuter ouvertement de ces exercices publics contribue aux fondements d'une société démocratique et nous sommes inquiets du fait que les acteurs ayant été sollicités et ayant participé à la CRPP refusent d’en discuter, même dans des conditions où nous nous engagions à respecter leur anonymat.

\section{RÉFÉRENCES}

Arsenault, J. (2014, 15 septembre). Révision des programmes : dialogue de sourds entre les syndicats et Québec. La Presse. Repéré à http://www.lapresse.ca/actualites/politique/ politique-quebecoise/201409/15/01-4800429-revision-des-programmes-dialoguede-sourds-entre-les-syndicats-et-quebec.php.

Arsenault, P.-É. (2016). Révision des dépenses publiques : s'agit-il d'un nouveau levier ou d'une simple panacée de circonstance? Dans M. Lamari et J. L. Jacob (éds.), Praxis de lévaluation et de la révision des programmes publics: approches, compétences et defis (pp. 85-114). Québec: Presses de l'Université du Québec.

Chouinard, T. (2014, 27 septembre). Un milliard de moins en éducation. La Presse. Repéré à http://www.lapresse.ca/actualites/education/201409/26/01-4804058-un-milliardde-moins-en-education.php.

Dutrisac, R. (2014, 24 novembre). Le prix de l'austérité : 2,3 milliards. Le Devoir. Repéré à http://www.ledevoir.com/politique/quebec/424752/le-prix-de-l-austerite-2-3milliards.

Gouvernement du Québec (2014a). Comptes publics 2013-2014. États financiers consolidés du Gouvernement du Québec, vol. 1. Repéré à http://www.finances.gouv.qc.ca/ documents/Comptespublics/fr/CPTFR_vol1-2013-2014.pdf. 
Gouvernement du Québec (2014b). Directive concernant l'évaluation de programme dans les ministères et les organismes. Extrait de la Gazette officielle du Québec (Décret 125-2014). Repéré à https://www.tresor.gouv.qc.ca/cadredegestion/actualites/detailde-la-nouvelle/directive-concernant-levaluation-de-programme-dans-les-ministereset-les-organismes/index.html.

Gouvernement du Québec (2014c). Rapport de la Commission de révision permanente des programmes. Repéré à https://www.tresor.gouv.qc.ca/fileadmin/PDF/revision_ programmes/rapport_2014.pdf.

Gouvernement du Québec (2015a). Rapport de la Commission de révision permanente des programmes. Cap sur la performance. Sommaire. Repéré à https://www.tresor.gouv. qc.ca/fileadmin/PDF/revision_programmes/rapport_2015_sommaire.pdf.

Gouvernement du Québec (2015b). Rapport de la Commission de révision permanente des programmes. Cap sur la performance. Volume 1. Les fondements d'un état performant et la mise en place d'un mécanisme de révision permanente des programmes. Repéré à https://www.tresor.gouv.qc.ca/fileadmin/PDF/revision_programmes/ rapport_2015_vol1.pdf.

Gouvernement du Québec (2015c). Rapport de la Commission de révision permanente des programmes. Cap sur la performance. Volume 2. La révision de certains programmes: quelques pistes. Repéré à https://www.tresor.gouv.qc.ca/fileadmin/PDF/revision_ programmes/rapport_2015_vol2.pdf.

Gouvernement du Québec (2015d). Rapport de la Commission de révision permanente des programmes. Cap sur la performance. Volume 3. Expériences hors Québec en matière de révision des programmes. Repéré à https://www.tresor.gouv.qc.ca/fileadmin/PDF/ revision_programmes/rapport_2015_vol3.pdf.

Gouvernement du Québec (2015e). Rapport de la Commission de révision permanente des programmes. Cap sur la performance. Volume 4. Les travaux de la Table sur l'aide financière au développement économique. Repéré à https://www.tresor.gouv.qc.ca/ fileadmin/PDF/revision_programmes/rapport_2015_vol4.pdf.

Gouvernement du Québec (2016). Comptes publics 2015-2016. États financiers consolidés du Gouvernement du Québec, vol. 1. Repéré à http://www.finances.gouv.qc.ca/ documents/Comptespublics/fr/CPTFR_vol1-2015-2016.pdf.

Gouvernement du Québec (2017). Stratégie de gestions des dépenses. Budget de dépenses 2017-2018. Repéré à https://www.tresor.gouv.qc.ca/fileadmin/PDF/budget_depenses/ 17-18/strategieGestionDepenses.pdf.

Groupe de travail sur l'examen des organismes gouvernementaux (1997). Rapport. Repéré à https://www.mce.gouv.qc.ca/publications/rapport_organismes_gouv_1997.pdf.

Jacob, J. L., Lamari, M., Poulin-Larivière, L. et Beaucage, J. (2016). Évaluation et révision des dépenses publiques : portrait analytique de l'expérience québécoise. Dans M. Lamari et J. L. Jacob (éds.), Praxis de l'évaluation et de la révision des programmes publics. Approches, compétences et défis (pp. 59-84). Québec : Presses de l'Université du Québec.

Lessard, D. (2014, 23 novembre). Commission Robillard : des économies possibles de 2,2 milliards. La Presse. Repéré à http://www.lapresse.ca/actualites/politique/politiquequebecoise/201411/23/01-4821688-commission-robillard-des-economies-possiblesde-22-milliards.php. 
Lessard, D. et Teisceira-Lessard, P. (2014, 3 septembre). Fonction publique : douloureuses compressions à prévoir. La Presse. Repéré à http://www.lapresse.ca/actualites/politique/ politique-quebecoise/201409/02/01-4796620-fonction-publique-douloureusescompressions-a-prevoir.php.

Lévesque, K. (2015, 26 novembre). Austérité ou rigueur budgétaire? La Presse. Repéré à http://plus.lapresse.ca/screens/4d57b632-f98a-46aa-b82e-f40b62b309f8\%7C6.Svb P56tr4a.html.

Nguyen, M. (2016). Bilan de l’observatoire sur les conséquences des mesures d’austérité 20142016. Document de réflexion. Institut de recherche et d'informations socio-économiquesIRIS. Repéré à http://iris-recherche.s3.amazonaws.com/uploads/publication/file/ Bilan_observatoire_WEB.pdf.

Organisation for Economic Cooperation and Development (2017). Spending review. Dans Government at a glance 2017. Paris : OECD Publishing. Repéré à www.oecd-ilibrary. org/governance/government-at-a-glance-2017_gov_glance-2017-en.

Parti Libéral du Québec (2014). Engagements. Parti Libéral du Québec. Élections générales 2014. Repéré à https://www.poltext.org/sites/poltext.org/files/plateformes/ plq2014.pdf.

Portail Québec (2015). Rapport de la Commission de révision permanente des programmes, Fil d'information. Repéré à http://www.fil-information.gouv.qc.ca/Pages/Article. aspx?idArticle $=2308318196$.

Secrétariat du Conseil du Trésor du Québec (2005). Rapport du Groupe de travail sur lexamen des organismes gouvernementaux : les 60 organismes désignés par le gouvernement pour 2004-2005. Repéré à https://www.tresor.gouv.qc.ca/cadredegestion/ fileadmin/documents/publications/sct/rapport_groupe_travail_2004.pdf.

Secrétariat du Conseil du Trésor du Québec (2006). Rapport du Groupe de travail sur l'examen des organismes gouvernementaux : les 58 organismes désignés par le gouvernement pour 2005-2006. Repéré à https://www.tresor.gouv.qc.ca/fileadmin/PDF/ publications/RapportGeoffrion2005-2006.pdf.

Secrétariat du Conseil du Trésor du Québec (2007). Rapport du Groupe de travail sur l'examen des organismes gouvernementaux : les 19 organismes désignés par le gouvernement pour 2006-2007. Repéré à https://www.tresor.gouv.qc.ca/cadredegestion/ fileadmin/documents/publications/sct/rapport_groupe_travail_2006.pdf.

Secrétariat du Conseil du Trésor du Québec (2013). Glossaire des termes usuels en mesure de performance et en évaluation. Pour une gestion saine et performante. Repéré à https:// www.tresor.gouv.qc.ca/fileadmin/PDF/publications/glossaire_termes_usuels.pdf.

Secrétariat du Conseil du Trésor du Québec (2017). Révision des programmes. Repéré à https://www.tresor.gouv.qc.ca/ministre-et-secretariat/revision-des-programmes/.

Smits, P. et Jacob, S. (2014). La fonction d'évaluation dans l'administration publique québécoise : analyse de la cohérence du système d'action. Administration publique du Canada, 57(1), 71-96. https://doi.org/10.1111/capa.12059

Teisceira-Lessard, P. (2014, 12 juin). L’ex-politicienne Lucienne Robillard révisera les programmes. La Presse. Repéré à http://www.lapresse.ca/actualites/politique/politiquequebecoise/201406/12/01-4775166-lex-politicienne-lucienne-robillard-revisera-lesprogrammes.php. 


\section{AUTHOR INFORMATION}

Marie-Pier Marchand est étudiante au doctorat en éducation à l'Université du Québec à Montréal. Dans le cadre de sa thèse, elle s'intéresse à la contribution des différents groupes de parties prenantes au sein des démarches d'évaluation. Elle s'est également impliquée dans la communauté évaluative afin de soutenir le développement et l'insertion des évaluateurs émergents et agit maintenant à titre de chargée de cours et d'assistante de recherche.

Astrid Brousselle est professeure et directrice de l'École d'administration publique à l'Université de Victoria. Elle a dirigé, entre 2011 et 2016, la chaire de recherche du Canada en Évaluation et amélioration du système de santé, à l'Université de Sherbooke. Son expertise principale porte sur les théories et méthodes évaluatives qu'elle applique au domaine de la santé et, plus récemment, à la question de la transition écologique. Astrid Brousselle est éditrice associée francophone de la Revue canadienne d'évaluation de programme. Elle a coédité et coécrit le livre Évaluation : Concepts et Méthodes (PUM, 2012). 